\title{
Milano, fin qui tutto bene: tra resistenza ed omologazione
}

\section{Milano, fin qui tutto bene: Between Resistance and Homologation}

EMANUELE Broccio [manuel_broccio@yahoo.it]

Université Paris X - Nanterre, Francia

\begin{abstract}
RiassunTo
Nel mio studio si intendono esplorare le modalità formali e le scelte tematiche di cui si serve Gabriella Kuruvilla per la rappresentazione di un ambiente multiculturale e delle relazioni che lo caratterizzano nel romanzo Milano, fin qui tutto bene (2012). Decostruendo puntualmente un certo diffuso immaginario, fondato sull'esaltazione degli effetti positivi di crescita e scambio offerti dalla mondializzazione, la narrazione, attraverso la strategia della focalizzazione multipla, lascia emergere una mappa del malessere, connotata dalla resistenza dei particolarismi culturali nel segno, spesso, di una conclamata conflittualità piuttosto che di un placido rapporto di assimilazione tra comunità etniche diverse. La prospettiva adottata, infatti, evidenzia un sentimento generale di paura ed ostilità verso gli "altri", ed una forma di paralisi sociale che investe quasi tutti i protagonisti, descritti come incapaci di integrarsi a fondo in qualsiasi relazione che metta in discussione le loro origini e radici. Il romanzo si inscrive nel circuito della letteratura mondiale, e più specificamente di quella categoria di testi isolati dalla critica sotto l'etichetta di "letteratura della migrazione", ed esplicita sia un deciso distanziamento dalla prospettiva eurocentrica sia la necessità di un ritorno alla realtà e al suo referente.
\end{abstract}

\section{PAROLe CHIAVE}

Multiculturale; Migrazione; Città; Focalizzazione; Realtà; Globalizzazione

\begin{abstract}
In this study I have explored the themes and language used by Gabriella Kuruvilla in her novel Milano, fin qui tutto bene (2012), focusing my attention on the representation of the multicultural context and the relations which take place within it. Koruvilla manages to shed light on the negative aspects of globalization through the narrative structure of multiple focalization, and maps a series of conflicts which regard the process of blending different cultures together. Feelings of fear and hostility towards the "others" and some sort of social paralysis inflict most of the characters who are often portrayed as incapable of creating deep bonds with anyone who threatens their roots or beliefs. The novel falls into the genre of global literature, more specifically into the group of texts labelled as "Migrant Literature", while showing an explicit distance from the Eurocentric perspective with a need for a return to reality and its objects.
\end{abstract}

\section{KEYWORDS \\ Multiculturalism; Migration; City; Focalization; Reality; Globalization}


Se la letteratura è coinvolta, al pari di vari campi del sapere, in quel vasto processo di partecipazione al fenomeno della globalizzazione ${ }^{1}$ e delle sue complesse conseguenze, sembra essere proprio la città - già topos di prestigiosa tradizione letteraria - il luogo testuale più appropriato attraverso cui misurarne la portata e gli effetti, a partire proprio dalla multiculturalità, facilmente riconoscibile come uno dei temi maggiormente frequentati da molti autori contemporanei. In tal senso, un contributo concreto alla costruzione di un immaginario letterario multiculturale ${ }^{2}$ è offerto da Milano, fin qui tutto bene, terzo romanzo della scrittrice italo-indiana Gabriella Kuruvilla (Laterza 2012), che, come suggerito dal titolo, ha per oggetto della narrazione proprio la metropoli lombarda.

Formalmente organizzato secondo la modalità della focalizzazione multipla (Genette 1972) in quattro sezioni - la cui istanza narrativa è affidata a altrettanti protagonisti-narratori intradiegetici i quali espongono lo scorrere della quotidianità milanese ciascuno dal proprio punto di vista - il testo è ulteriormente scandito da un indice onomastico che indica con precisione i quartieri dentro i quali, di volta in volta, si svolge l'azione. La scansione è, inoltre, suggestivamente sottolineata dal corredo iconografico di Silvia Azzari tramite quattro foto, disposte in apertura di ogni capitolo, che rafforzano anche visivamente la geometrica ripartizione narrativa. Attraverso i racconti di Anita/Paola, Samir, Stefania e Tony, le cui storie incrociano appena l'una il sentiero dell'altra con il pretesto della vendita di un letto a soppalco Ikea e della restituzione di un paio di stivali, il lettore è introdotto e condotto tra le strade di Via Padova, Viale Monza, Sarpi e Corvetto. Strategica, quindi, la funzione prolettica dell'indice onomastico che, sopperendo all'ambiguità del titolo, informa il lettore sulle aree cittadine in cui sta per addentrarsi: frazioni periferiche e popolari, lontane dalla Milano da bere, e note come territorio di convivenza, in alcuni casi conflittuale, di diverse comunità etniche.

È la stessa autrice a dichiarare che il modello ripartito del punto di vista le sarebbe stato suggerito dalla visione di Babel, pellicola di successo internazionale del regista brasiliano Inarritu ${ }^{3}$. Tuttavia, secondo la critica "la proliferazione delle voci e dei punti di vista nel racconto è da considerare uno dei lasciti testamentari del postmoderno, transitati [...] nella narrativa italiana

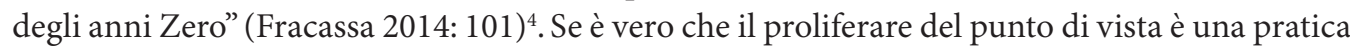
di ascendenza squisitamente letteraria, è tuttavia innegabile che l'esplicito riferimento di Kuruvilla a un'opera cinematografica descrive e dà conto appieno della temperie culturale da cui scaturiscono molte opere letterarie oggi, sempre al centro - al tempo della globalizzazione - di

1 Per una riflessione organica su questa relazione, che ha raccolto le problematiche emerse all'interno del dibattito accademico, sistematizzandole in uno studio meritevole si rimanda a Benvenuti - Ceserani (2012). Complementare al volume dei due studiosi, offrendo nel mentre casi di studio che, in fase analitica, verificano l'effettiva portata dei postulati teorici, è il volume della rivista Narrativa (Contarini et al. 2014).

2 A proposito della tassonomia rilevata intorno alla multiculturalità, all'interno di una riflessione di ben più ampio respiro sulla letteratura migrante, si rimanda al dettagliato lavoro Narrazioni contese. Vent'anni di scritture italiane della migrazione (Mengozzi 2013). Sulla multiculturalità, cfr. soprattutto le pp. 68-72.

3 In un'intervista consultabile su youtube.com, Kuruvilla afferma: "Un romanzo dove ci sono quattro personaggi principali che vivono in queste quattro zone, che le raccontano dal loro punto di vista, quindi anche quattro visioni molto diverse sulla città [...] questi quattro personaggi si incrociano perché, in realtà, si vogliono scambiare un soppalco Ikea, come nel film Babel di Inarritu”. Le citazioni tratte da questa intervista sono trascritte da chi scrive.

4 La genesi di tale pratica, tuttavia, potrebbe essere anticipata e ascritta all'esperienza letteraria di quegli scrittori per i quali la critica ha coniato la categoria di Modernismo. 
diversi linguaggi artistici, tra i quali il cinema occupa di certo un posto privilegiato, offrendosi sempre più spesso, come in questo caso, in qualità di "ipotesto". Si invertono così i ruoli di un dialogismo storicamente consolidato in passato intorno alla Letteratura come originaria fonte di ispirazione dell'arte cinematografica. Un mutamento tutt'altro che compiuto e definitivo, se si pensa a quella che potremmo chiamare oggi l'angoscia dell'influenza digitale, data l'importanza sempre maggiore e inarrestabile assunta negli ultimi tre decenni dalle fonti digitali e dalla loro primaria responsabilità nel fenomeno della globalizzazione.

Questa marginale contraddizione, relativa alla genesi del romanzo, tra la dichiarazione autoriale e quanto invece sostenuto dalla critica, non è che la prima di numerose altre con cui lo studioso, ma anche il lettore comune, si confronterà in fase ermeneutica, all'interno di un sistema di segni testuali frammentario e, spesso, poco coerente.

La scelta formale della focalizzazione multipla è rafforzata da una diversificazione linguistica che tende, mimeticamente, a riprodurre, differenziandolo di volta in volta, il linguaggio della vita quotidiana: la "plurivocità", per dirla con Bachtin, coincide con la plurietnicità ${ }^{6}$ I personaggi vengono, infatti, connotati da una marcata caratterizzazione linguistica e culturale tramite un interessante impianto ideativo: Anita (Paola), meticcia italo-indiana e, limitatamente, alterego della scrittrice, si esprime attraverso una sequela di proverbi popolari, riportati in forma di frammenti di monologo interiore; il linguaggio di Samir è inframezzato da espressioni in lingua araba; uno spiccato milanese sostiene i pensieri di Stefania; un codice linguistico, infine, ibrido tra dialetto napoletano, milanese, e slang giamaicano illustra le pittoresche giornate di Tony. Nel complesso, tuttavia, non si tratta di un'operazione felicemente riuscita, e basti pensare ai motti popolari di Anita, durante il suo peregrinare per Via Padova, che risultano per lo più estranei rispetto al contesto in seno al quale vengono esperiti e che vorrebbero in qualche modo descrivere, seppur con funzione antifrastica. All'interno dell'accentuata diversificazione terminologica, la lingua, poi, è caratterizzata, in modo omogeneo, da un andamento sintattico leggero, di tipo paratattico, modulato sugli stilemi dell'idioma parlato. "Un ritmo sincopato" commenta l'autrice, simile a quello dell'hip hop e del rap, musiche da lei amate e ascoltate. Un linguaggio, quindi, volutamente collocato dentro la realtà contemporanea, improntata a ritmi rapidi, una dimensione in cui tutto scorre velocemente.

Tuttavia, la presenza di un'accentuata diversificazione terminologica caratterizzante le quattro voci narranti e l'estrema omogeneità della costruzione sintattica, dietro la quale è facilmente riconoscibile una specifica ed unica voce autoriale, creano un conflitto insanabile e assottigliano il preteso sistema polifonico, riducendo notevolmente nel testo il dinamismo sotteso all'intreccio di differenti voci. Una contraddizione che ne suggerisce ancora un'altra. A questo stile rapido, infatti, che illustra in modo conciso ma minuzioso luoghi, eventi, stati emotivi, passando spesso in modo scorrevole (e con notevole abilità di manovra di tanto materiale, all'interno dello stesso spazio narrativo) da un contesto all'altro, si oppone la stasi dei personaggi che vi si muovono. Dietro la centralità dei luoghi descritti, spesso in un sovraccarico di informazioni

$5 \quad$ Il riferimento è al noto studio sull'intertestualità in ambito poetico. Vedi Bloom (1983).

6 A questo proposito vedi Fracassa (2014: 110-11). Lo studioso in apertura del suo contributo spiega i termini entro cui parlare di letteratura dell'Italia multiculturale, "dicitura della quale, peraltro, non si intende sottovalutare [...] la potente eco continiana" (101).

$7 \quad$ Intervista a Kuruvilla, cit. 
e dettagli, nel caos delle esistenze che popolano quelle strade, si celano infatti un largo anonimato e una forma di reticenza dei protagonisti a prendere davvero parte a quella vita. Il segno più esplicito della loro inerzia è l'assenza di una reale interazione all'interno delle relazioni narrate. Ogni rapporto è sospeso sulla soglia di una conoscenza superficiale in cui si guarda all'altro come a un diverso da cui difendersi, o al quale approcciarsi con prudenza: una prudenza che assume spesso le sfumature della riluttanza per sfociare poi in vera e propria diffidenza. Esemplare, a tal proposito, l'incontro di Paola, una mattina come tante, con una ragazza rom a cui si accompagna durante il resto della giornata ma della quale non si fida affatto, sospettando sempre in ogni luogo in cui si trovano che lei possa rubare, o addirittura abbandonarsi ad atti di vandalismo, proiezione chiarissima delle sue paure, che per traslato si fanno espressione delle paure diffuse e condivise da un certo immaginario collettivo, secondo cui rom è sinonimo necessariamente di delinquenza e criminalità:

Ci fermiamo davanti a Cargo. "Vieni con me", mi dice. Almeno non l'aspetto fuori [...] Non capisco cosa stiamo facendo. Se ruba qualcosa me ne vado, penso [...] "Vieni con me", mi dice. Non sto facendo altro. Usciamo dal negozio. La seguo lungo una via, oltre un cancello, dentro un giardino: temo voglia entrare in un appartamento. La immagino che spacca una finestra, scavalca il davanzale, gira per le camere, prende degli oggetti, sputa per terra, mi urla “Scappiamo!”. E invece mi accorgo che al piano terra di questa villa del '700 c’è un bar, aperto a tutti: colonne in pietra, arredo in legno, sedie in paglia, quadri pop e grandi finestre. (Kuruvilla 2012: 24-25)

Il sentimento di paura latente non scaturisce, tuttavia, esclusivamente dalla diversità etnica, ma coinvolge qualsiasi forma di distanza sociale, come si evince dai pensieri di Stefania in preda al panico in casa di Tony mentre recupera i suoi stivali.

Gli spazi descritti in questo romanzo sfumano l'immaginario di una contrapposizione, spesso anacronistica, tra campagna e città, tramite la rappresentazione della vita di strade periferiche che occupano ora lo spazio intermedio tra le due storiche polarità. Tuttavia, l'idea di vie popolari vissute come salotti ${ }^{8}$ in cui la gente si riversa per discutere, camminare, lavorare, amare, morire recuperando - in fin dei conti - l'antica funzione della polis, è delusa da specifici segni testuali che restituiscono un quadro della realtà improntato a un modello di divisione piuttosto che di scambio e integrazione. Un luogo nel quale le uniche interazioni che sfociano nella fusione, in una relazione che superi il confine della diversità etnica, religiosa, culturale tout court e che sembrano destinate a ripetersi, e quindi a persistere, sono quelle legate alla sfera dell'abiezione e dell'illecito come nel caso della prostituzione giovanile omosessuale:

Non sono una bacchettona, è la disperazione insita in questo mercificio di corpi vecchibianchi e giovanineri ad intristirmi. Meglio l'apartheid dei rapporti, piuttosto. (Kuruvilla 2012: 9)

Dove interessante è notare l'aspetto grafico di vecchibianchi e giovanineri, resi tramite un'unica parola, senza separazione tra il sostantivo e l'aggettivo, neologismo coniato per indicare una

8 "Come fanno adesso gli immigrati che in realtà adesso rivivono la strada come se fosse un salotto." Intervista a Kuruvilla, cit. 
vera e propria categoria che risulta così ribattezzata. Il resto delle relazioni resta invischiato in rapporti formali che non riescono a superare la soglia della diversità. Ne è dimostrazione la concezione del rapporto amoroso tra individui di comunità etniche diverse, che emerge dalle storie sentimentali di questi personaggi. Così Paola/Anita descrive il padre di suo figlio:

Il suo Edipo era un ragazzo bello e ricco e innamorato, in pratica lo stereotipo delluomo ideale. "Nessuno è perfetto", avrebbe detto mia madre. Si sbagliava: lui lo era. E non potendo sopportare il senso di inferiorità che mi procurava, l'ho lasciato. Dicendogli che amavo un altro, anche se non era vero: ma era l'unico modo per non vederlo più tornare. Prima, però, l'ho baciato. "Un bacio tira l'altro e si finisce in sala parto”, avrebbe detto mia madre. Così è stato. (Kuruvilla 2012: 18)

E ancora Sarmi, mentre rievoca il momento di rottura con la sua fidanzata:

Ridendo, le ho chiesto: “Ma sei incinta?”. "Sì: è tuo”, mi ha risposto. Mi sono alzato dalla panchina e sono andato da lei. "Stai scherzando?", le ho domandato. "Mai stata così seria”, mi ha risposto. "E vuoi tenerlo?”. “Sono oltre il quinto mese: non potrei abortire neanche volendo e comunque non voglio" “Tu sei pazza”. "E tu sei uno stronzo", mi ha detto. "Sicuramente”, ho ribattuto. "Io voglio stare con te". "Io non penso". "Non pensi o non vuoi?", mi ha chiesto. "Ci penso", le ho risposto mentre me ne andavo. “Te ne pentirai”, mi ha urlato. Ma'lesh: no problem. Meglio l'incertezza del rimpianto che la certezza del rimorso, ho pensato (Kuruvilla 2012: 59)

E se, nel caso di Paola, le ragioni della sua rinuncia sono esplicitate, possiamo supporre con un buon grado di approssimazione che sia sempre la diversità culturale, rafforzata dal divario economico, a impedire a Samir di essere felice, abbandonandosi al sentimento amoroso, sebbene nel testo non ci sia detto nulla sulle sue motivazioni. Non è un topos inedito, e sarebbe interessante, all'interno della riflessione critica sulla letteratura della migrazione, valutare come, a partire da La straniera, i rapporti amorosi tra personaggi appartenenti a culture differenti ${ }^{9}$ sembrano essere per lo più destinati al fallimento ${ }^{10}$. Così, la rinuncia dei protagonisti di Milano fin qui tutto bene alla possibilità di amare ed essere felici è speculare della loro rinuncia a una reale integrazione, della loro incapacità di guadagnare attraverso la relazione amorosa - che è la più intima, e pertanto la più esposta tra le relazioni umane - una nuova esistenza. Emblema di una radicale mancanza di coraggio, il voluto fallimento del rapporto a due è il manifesto dell'impossibilità di affondare in una esistenza completa, costruita sull'opportunità di confronto e assimilazione reciproci tra le proprie origini e quelle del partner.

9 È vero che ne La straniera i due protagonisti sono entrambi di origine araba, tuttavia è profondamente diversa la loro condizione sociale: agiata e, per certi versi, profondamente omologata al modello occidentale quella dell'architetto; povera e subalterna quella di Amina che rappresenta un caso esemplare di emarginazione. Sul romanzo di Tawfik, all'interno della vasta bibliografia, si rimanda soprattutto a Felici (2005) e Contarini (2012).

10 In modo lungimirante, anche Chiara Mengozzi, analizzando I sessanta nomi dell'amore, sottolinea come l'impossibilità per i due protagonisti di concretizzare il rapporto amoroso dipenda proprio da un cortocircuito comunicativo, da un problema di traduzione culturale: "la traduzione, infatti, è innanzitutto ciò che costruisce e decostruisce la relazione amorosa tra i due protagonisti, ovvero ciò che la rende (im)possibile, e in secondo luogo è ciò che innesca la pratica stessa della scrittura alimentando il desiderio, che è insieme desiderio dell'altro, desiderio di traduzione dell'altro." (Mengozzi 2013: 174). 
La resistenza manifestata dai protagonisti, svuotata di quei valori che generalmente la sostengono, si riduce in una sorta di passiva accettazione del discrimine. Incapaci di spezzare la crosta della diversità, cogliendo l'attimo che rende meravigliosa l'esistenza, questi personaggi restano intrappolati nella categoria della non azione, il che sbalordisce quando il lettore confronta il loro operato con la pretesa dinamicità dei luoghi descritti che sono teatro di quelle azioni ${ }^{11}$.

Ma quali e come sono realmente i luoghi tracciati da Kuruvilla? La realtà più vicina ai quartieri che fanno da sfondo alle diverse vicende narrate è quella del ghetto. Come i ghetti, i quartieri di Milano fin qui tutto bene sono dotati di una duplice accezione: di luogo pericoloso, ma anche di steccato sicuro, di recinto entro cui il grosso gregge della diversità viene protetto dal resto del mondo. Nel primo caso, basti pensare all'inserimento di fatti di cronaca reale che, intrecciandosi con le storie finzionali, tendono a delineare un contesto con le proprie leggi, sottratte a quelle dello Stato, dell'ufficialità e della cosa pubblica, alle quali si ribellano fino allo scontro aperto. Emblematica, invece, della seconda accezione è la vicenda di Anita, a proprio agio solo in Via Padova, e assalita da un senso di spaesamento ${ }^{12}$ in qualsiasi altro luogo.

Una perfetta sintesi, infine, del duplice significato assunto dal ghetto è individuabile e sintetizzabile nella ricorrente espressione "Corvetto comanda" che contraddistingue la "storia" di Tony. Ripetuta come una sorta di mantra quotidiano con senso di orgogliosa appartenenza, la frase implica da un lato il sistema di regole interne e il pericolo che corre qualsiasi trasgressore; dall'altro il perimetro chiuso di un luogo che rende forti e sicuri i suoi abitanti, mettendoli in salvo dalla minaccia del mondo esterno.

La trama narrativa, cosi condotta, suggerisce una riflessione di più ampio respiro sul tema della identità oggi, esibendo una tipologia di personaggi che, oltre ad aver perso il ruolo di soggetti agenti, esplicitano la frattura e la definitiva crisi dell'io. Una condizione limbica di perdita di ogni punto di riferimento e della percezione del sé, riconoscibile, in apertura del testo, attraverso la frantumazione e lo sdoppiamento di Paola/Anita:

Via Clitumno 11, citofonare Paola Rossi”. Tanto il citofono non funziona, è stato sradicato. Tanto non mi chiamo Paola Rossi, ma Anita Patel. Quindi gli ho dato quel biglietto, sapendo che non riuscirà a scovarmi. Non ho alcuna voglia di rivederlo, forse (Kuruvilla 2012: 3)

Il pensiero della protagonista - posto in una posizione incipitaria di cui il lettore non può non tener conto - reca con sé i segni di un fallimento radicato e irrimediabile: da una parte la rinuncia ai natali stranieri, evidenti nel cognome Patel, dall'altra l'assunzione di un'identità ita-

11 Sull'isolamento, invece, delle quattro sezioni narrative e della mancanza di un reale nesso interno, riprendiamo ancora le osservazioni di Ugo Fracassa che, rifacendosi al giudizio artistico di Francesca Della Monica sulla produzione pittorica della scrittrice, scrive: "Gabriella Kuruvilla è anche un'artista ed è interessante notare nella sua produzione visuale la stessa attitudine separativa, in immagini caratterizzate da colori nettamente definiti e da figure isolate e disposte saldamente in griglie" (Fracassa 2014: 103).

12 A tal proposito, sembra che il ritorno di questo sentimento - difficilmente ascrivibile alle dinamiche della casualità - vada inteso come l'intenzionale citazione intertestuale dell'omonimo romanzo di Vasta, che, come noto, ruota intorno al racconto della città di Palermo e dei suoi cambiamenti. L'eco vastiana si esplicita ancor di più nell'episodio di Stefania che, proprio come il protagonista del romanzo dello scrittore siciliano, deve fare i conti con il rapido mutamento dei luoghi cittadini: "solo che adesso mi sento spaesata: sono diversa io, ed è diversa la gente che lo frequenta. È diversa tutta questa zona, purtroppo” (Kuruvilla 2012: 75). 
lianissima, inscritta invece nel cognome Rossi, che serve però solo per nascondersi e non essere scovata; nessuna speranza, quindi, di un riscatto dalla condizione di rassegnato isolamento in cui il personaggio ha scelto di relegarsi.

Non mancano, poi, sottili osservazioni autoriali, celate dietro alcuni commenti dei protagonisti, in cui è ravvisabile una vera e propria critica al concetto di identità:

Sto nello sprofondo, direbbero a Roma: che per me che sono nata a Milano vuole dire oltre il fondo, più o meno. (Kuruvilla 2012: 3)

L'espressione romana è descritta come accessibile esclusivamente nel suo significato letterale a una milanese nativa, marcando che il concetto di diversità non riguarda solo la multietnicità, ma si estende anche alle differenze esistenti all'interno dello stesso paese tra zone diverse perché allude anche al superamento dell'anacronistica equivalenza nazione/identità. Osservazioni sottili come questa rivelano la maturata consapevolezza della scrittrice intorno al dibattito critico sulla specificità della letteratura oggi e del suo complesso rapporto con i processi di mondializzazione, all'interno dei quali i movimenti migratori e i problemi della convivenza multietnica sono certamente tra le tematiche più discusse. Da questo punto di vista, la scelta di un contesto multiculturale, nelle forme emerse finora dalla nostra lettura, legittima l'allineamento del romanzo, tanto dal punto di vista formale quanto da quello tematico, alla categoria di testi isolati dalla critica come "letteratura della migrazione", caratterizzati, tra gli altri aspetti, da un marcato "ritorno" alla realtà e al referente, tendenza per altro diffusa anche nella letteratura italiana tout court $^{13}$. Oltre alla costruzione di uno sfondo narrativo caratterizzato dall'inclusione di reali fatti di cronaca, soprattutto nell'episodio di Corvetto, l'aspirazione a un realismo ancora più denso e credibile si traduce nei riferimenti a Saviano, citato esplicitamente, e nel parallelismo tracciato tra i quartieri di Corvetto e di Scampia, come avviene all'interno dell'ultimo racconto:

Siamo passati davanti al Saverio's caffè: il bar di un ecuadoregno che, naturalmente, si chiama Saverio. È nato in Ecuador e si è trasferito a Milano quindici anni fa: è uno che dell'Italia ama il calcio e la letteratura. E infatti, nel suo locale, sulle pareti e sui ripiani, mette in mostra le bandiere dell'Inter e i libri di Saviano come se fossero suoi personali trofei. (Kuruvilla 2012: 61)

Il primo a trasferirsi a Corvetto è stato mio padre, poi l'abbiamo raggiunto io, mia madre e mia sorella e alla fine ci ha seguito pure mio nonno. Arriviamo tutti da Scampia, ma qui ci sentiamo a casa: questo quartiere, con Scampia, è pure gemellato. Ovvio che ci sta il feeling con la gente del posto: qua sta pieno di terroni. (Kuruvilla 2012: 100)

Questo procedimento - insieme alla già citata eco vastiana a proposito dello spaesamento - suggerisce l'idea di un romanzo che intenzionalmente si contamina col genere saggistico, esprimendo il proprio punto di vista su diverse problematiche sociali relative alla contemporaneità. Mondializzazione e multiculturalità, nel micro-modello cui rimanda la rappresentazione

13 Sul problema del realismo in letteratura vedi almeno Siti (2013), il numero di Allegoria completamente dedicato al tema (Donnarumma et al. 2008) e Antonello, Mussgnug (2009). 
narrativa di Kuruvilla, non sembrano offrire segni di speranza in un eventuale processo di assimilazione o di svolta dello stato delle cose, per il quale manca qualsiasi presupposto o volontà. Significativi, a questo proposito, l'inizio e la fine di ogni episodio narrativo che, con modalità circolare, si innestano sul solitario isolamento dei protagonisti i quali lungo la narrazione sfiorano appena l'esistenza dell'altro senza mai attraversarla del tutto, proteggendosi da ogni rischio ma perdendo, nel contempo, l'opportunità di vivere. Milano fin qui tutto bene, allora, demistifica anche qualsiasi immaginario di mondializzazione esaltata nei suoi effetti positivi di crescita e scambio, e lascia emergere una mappa del malessere, connotata dalla resistenza dei particolarismi culturali nel segno, quindi, di una conclamata conflittualità piuttosto che di un placido rapporto di assimilazione. Un realtà in bianco e nero, proprio come nelle quattro foto poste in apertura di ogni capitolo che ne sono corredo iconografico.

Sottilmente allusivo, in tal senso, è il titolo del testo: il sintagma "fin qui tutto bene" contiene in sé un'accezione negativa, e solo in minor misura antifrastica ${ }^{14}$, per indicare come, oltre la stasi del momento descritto, nulla garantisce il mantenimento pacifico dello stato di cose, trattandosi anzi di un equilibrio apparente e precario perché realizzato per lo più sulla mancata esposizione al confronto, e pertanto aperto a probabili conflittualità. Implicitamente, si ricava che lo scambio e l'interazione sociali sono giudicati alla stregua della rimozione delle proprie peculiarità culturali - quelle caratteristiche che ci rendono diversi ma unici gli uni rispetto agli altri - e non come l'opportunità di una loro completa accettazione, da considerarsi concretamente in atto solo a patto del confronto e dell'accettazione delle diversità altrui.

Uno dei segni testuali intorno a cui si addensano ulteriori cause dell'inazione dei personaggi è il frequente ricorso alla contrapposizione tra il presente ed il passato. Alla dettagliata descrizione odierna dei luoghi presentati, infatti, fa seguito la puntuale rievocazione del passato, la sostituzione delle attività commerciali italiane con quelle straniere:

Sparivano il calzolaio, la latteria e il barbiere ma sulle loro ceneri come fenici aprivano i fruttivendoli e gli internet point cingalesi, i ristoranti e gli alimentari sudamericani, le macellerie e i kebab arabi oltre, ovviamente, alle molte attività dei cinesi, che non gestiscono solo parrucchieri ma anche negozi d'informatica pieni di cellulari e computer, centri-massaggi che quasi tutti pensano siano bordelli e anche incasinatissimi bazar, che vendono dalle parrucche, da utilizzare se il parrucchiere a otto euro non ha fatto un ottimo lavoro, fino ai casalinghi e all'abbigliamento. (Kuruvilla 2012: 13-14)

La moltiplicazione produttiva capitalistica, derivazione diretta del processo di globalizzazione, viene, poi, indicata, fin dalla sua nascita, come responsabile del fallimento delle piccole aziende e, implicitamente, di una inevitabile de-valorizzazione delle merci:

Erano gli anni del boom dei supermercati, quelli: tutti a far scivolare il carrello tra uno scaffale e l'altro, ascoltando musica pop abbagliati da lampade al neon. In preda a un istinto bulimico, vagavamo con la lista della spesa in mano, neanche fosse una bussola capace di orientarci, dentro il

14 Secondo Fracassa (2014: 105) il titolo del romanzo è da ricondurre al film La Haine, e sarebbe una citazione delle parole pronunciate dal protagonista poco prima di schiantarsi al suolo "L'important ce n'est pas la chute ... c'est l'atterrisage". 
paradiso dei balocchi per adulti: che nasceva sui cadaveri delle fabbriche occupandone lo spazio e uccideva l'esistenza dei negozi al dettaglio rubandone i clienti. (Kuruvilla 2012: 13)

Una prospettiva sottilmente eurocentrica cui Kuruvilla oppone, all'interno di un'altra sezione narrativa, la denuncia fatta da Samir sulla durezza delle condizioni di vita esperite dagli immigrati, nel segno della perdita di qualsiasi coordinata esistenziale:

Altro che diffidenza: noi che siamo arrivati in Italia dalla fine degli anni '90 in poi siamo trattati come rifiuti gettati dal mare sulle coste e veniamo lasciati in attesa di essere smaltiti altrove. Ma poi, altrove, non sempre riusciamo ad andare e a volte restiamo qui, in quello che cè il rischio che rimanga sempre un altrove: makan tani. Altrove rispetto a noi. (Kuruvilla 2012: 55)

Nel nome, poi, di una precisa scelta autoriale modellata su un sistema che - in egual misura dà voce a punti di vista contrastanti, privandoli tuttavia di una sintesi dialettica e senza esimerli dal rischio della stereotipizzazione, la critica, estesa alla sfera lavorativa, denuncia una rete di soprusi che coinvolgono insieme alla manodopera immigrata anche quella italiana, additando, non a caso, un padrone cinese come feroce simbolo del potere economico:

Così noi subiamo, wa noskut: e stiamo zitti. L'unica volta che ci siamo lamentati chiedendo al capo di metterci totalmente, e non parzialmente, in regola, ci ha risposto: "Dietro di voi cè la fila". Lui, il capo, è un cinese piccolo, brutto e cattivo: piscinin, brut e cativ, dite voi. E ha pure la puzza sotto il naso: è convinto che Pechino sia il centro del mondo, altro che Milano, figuriamoci il Cairo. (Kuruvilla 2012: 60)

A livello più intimo, questa contrapposizione presente-passato è rafforzata dall'associazione del passato alla sfera del rimpianto, come se la possibilità di vivere e di partecipare - seppur per un attimo - a una qualche felicità sia esclusivamente ancorata a ciò che è stato, e quindi irrimediabilmente perduto. È nel passato infatti che Anita/Paola e Samir vivono, rispettivamente, le loro storie d'amore, narrate con procedimento analettico, ed è soprattutto nei luoghi simbolo del suo passato che si rifugia Samir nei momenti onirici in cui proietta nel giardino di casa dei suoi genitori, in Egitto, l'immagine di sé mentre gioca con il figlio:

Non vorrei rivedere lei, vorrei solo rivedere mio figlio [...] Andare alla biglietteria etnica Amrun Travel e comprare un volo per due Milano-Cairo. Presentarlo a mia madre e mio padre. Insegnargli a lanciare i piatti, pieni di cibo mica di avanzi, al di là del muro che recinta il giardino di casa mia. Che poi sarebbe anche casa sua. Insha-allah: se Dio vuole. (Kuruvilla 2012: 59-60)

La memoria, allora, diviene il mezzo grazie al quale costruire un altrove, inevitabilmente idealizzato, in cui tutto sembrava potesse ancora accadere.

La tipologia caratteriale dei protagonisti che popolano le pagine di questo romanzo, appiattita sullo stereotipo, è sostenuta - su un piano diverso - dal sistema dei personaggi secondari, anch'esso costruito su una precisa omologazione. In tal senso, basterà richiamare brevemente l'attenzione su Gioia e Pietro: la prima, personaggio che deve la propria fama alla bellezza; il 
secondo - suo fidanzato - regista di documentari sugli immigrati, è di fatto mostrato come schiavo delle droghe e del desiderio di ottenere Samir, di cui si dice innamorato, sebbene lo abbia incontrato solo due volte. I loro profili sono costruiti sulla superficialità dei caratteri di una certa società, quella contemporanea, del consumo e dello spettacolo, in cui l'essere si assottiglia nell'apparire.

Tanto i protagonisti, con la resistenza all'integrazione, quanto i personaggi secondari, omologati a certe tipologie di cui il lettore riconosce subito i margini, sollecitano a inscrivere questo testo all'interno di quella categoria di opere contemporanee suggestivamente definita da Timothy Brennan Third-World Metropolitan Fiction (1997), da Damrosch Global Novel (2003) o da Coletti Romanzo mondo (2011) ${ }^{15}$. Nel testo di Kuruvilla, infatti, sono presenti molte delle caratteristiche indicate da quest'ultimo come tipiche dei romanzi

che sono stati, in un primo tempo, percepiti come non appartenenti ad una cultura nazionale specifica e quindi in qualche modo ricevibili da tutte le culture, e, poi, direttamente orientati $\mathrm{o}$ addirittura programmati per un lettore mondiale, cioè non legato a una cultura nazionale particolare [...] opere molto traducibili che ambiscono al mercato globale, desideroso di acquistare prodotti standardizzati e ben padroneggiabili, ma conditi di sapori locali. (Coletti 2011: 9)

Allo stesso modo, nel romanzo di Kuruvilla, ritroviamo una serie di tematiche che gli consentono di imporsi facilmente all'attenzione e alle tendenze di un pubblico globalizzato di cui assecondare le aspettative.

All'interno di un dispositivo narrativo così strategicamente costruito, Milano fin qui tutto bene non si sottrae al compito della letteratura impegnata, sollecitando una serie di riflessioni sulla realtà da cui parte, e promuovendo il superamento del carattere autoreferenziale della stessa letteratura, a favore del suo contributo ad un circuito interdisciplinare che si occupa di alcune delle questioni più urgenti della contemporaneità.

\section{Riferimenti bibliografici}

Antonello, P., \& Mussgnug, F. (Eds.). (2009). Postmodern Impegno. Ethics and Commitment in contemporay Italian Culture. Oxford: Peter Lang.

Benvenuti, G. (2012). Il romanzo neostorico italiano. Storia, memoria, narrazione. Roma: Carrocci.

Benvenuti, G., \& Ceserani, R. (2012). La letteratura nelletà globale. Bologna: Il Mulino.

Bloom, H. (1983). L'angoscia dellinfluenza. Milano: Feltrinelli.

Brennan, T. (1997). At Home in the World. Cosmopolitanism Now. Cambridge: Harvard University Press.

Contarini, S. (2012). Ricomposizioni identitarie: genere, etnia e classe in La straniera di Younis Taw-

fik. Italogramma [online], 2. In: http://epa.oszk.hu/02300/02391/00002/pdf/EPA02391_italogramma_02_2011_243-254.pdf

15 Damrosch, nel suo fondamentale What is World Literature (2003), distingue tra World Literature (connotata positivamente come "declinata localmente e mobile translocalmente") e Global Literature (omologata, standardizzata ed eventualmente infarcita di esotismo). L'uso che Coletti fa dell'espressione Romanzo mondo è in parte ispirato ma sostanzialmente diverso dalla proposta di Moretti (2003). 
Contarini, S., Marras, M., Pias, G., \& Quaquarelli, L. (Eds.). (2014). La letteratura italiana al tempo della globalizzazione. Numero monografico di Narrativa. Nuova serie, 35-36.

Coletti, V. (2011). Romanzo Mondo. La letteratura nel villaggio globale. Bologna: Il Mulino.

Damrosch, D. (2003). What is World Literature. Princeton-London: Princeton University Press.

Donnarumma, R., Policastro, G., \& Taviani, G. (2008). Ritorno alla realtà? Narrativa e cinema alla fine del postmoderno. Numero monografico di Allegoria, 57.

Felici, I. (2005). Régard croisés sur l'immigration marocaine en Italie. La straniera de Younis Tawfik. Babel, 2, 255-86.

Fracassa, U. (2014). Globalizzazione all'indice: modelli macrotestuali nella narrativa dell'Italia Multiculturale. Narrativa. Nuova serie, 35-36, 101-111.

Genette, G. (1972). Figure III. Discorso del racconto. Torino: Einaudi.

Intervista a Gabriella Kuruvilla. In: https://www.youtube.com/watch?v=xBhMJV3BH64.

Mengozzi, C. (2013). Narrazioni contese. Vent'anni di scritture italiane della migrazione. Roma: Carocci.

Moretti, F. (2003). Opera mondo. Torino: Einaudi.

Siti, W. (2013). Il realismo è l'impossibile. Roma: Nottetempo. 
